Canad. Math. Bull. Vol. 22 (3), 1979

\title{
ON SURFACES OF ORDER THREE
}

\author{
BY \\ TIBOR BISZTRICZKY
}

A surface of order three $F$ in the real projective three-space $P^{3}$ is met by every line, not in $F$, in at most three points.

In the present paper, we determine the existence and examine the distribution of elliptic, parabolic and hyperbolic points; that is, the differentiable points of $F$ which do not lie on any line contained in $F$.

We define a topology of $P^{3}$ in the usual manner. We denote the planes, lines and points of $P^{3}$ by the letters $\alpha, \beta, \ldots ; L, M, \ldots$; and $p, q, \ldots$ respectively. For a collection of flats $\alpha, L, p, \ldots ;\langle\alpha, L, p, \ldots\rangle$ denotes the flat of $P^{3}$ spanned by them. For a set $M,\langle M\rangle$ denotes the flat of $P^{3}$ spanned by the points of $M$.

1. A (plane) curve $\Gamma$ is the union of a finite collection of sets $C_{\lambda}(M)$ where the $C_{\lambda}$ 's are continuous maps from a line $M=\left\{m, m^{\prime}, \ldots\right\}$ into a plane $\alpha$. Let $p \in \alpha$. Then $p$ is a simple [double] point of $C_{\lambda}(M)$ if the equation $p=C_{\lambda}(m)$ has exactly one solution [exactly two solutions] $m \in M$.

Let $C=C_{\lambda}$. The line $T_{m}=\lim \left\langle C(m), C\left(m^{\prime}\right)\right\rangle$, as $m^{\prime} \neq m$ tends to $m$, is the tangent of $C$ at $m$. Let $C$ be differentiable; that is, $T_{m}$ exists and $\left|T_{m} \cap C(M)\right|<$ $\infty$ for every $m \in M$. We introduce (cf. [1] 1.3 .3 and [4]) the characteristic of $C$ at $m$ and the multiplicity with which a line $L \subset \alpha$ meets $C$ at $m$. Then $C$ is of order $n$ if $n$ is the supremum of the number of points of $M$, counting multiplicities, mapped into collinear points by $C$.

If $C$ is of order two [three], we denote $C(M)$ by $S^{1}\left[F_{*}^{1}\right.$ ]. Every point of an $S^{1}$ is simple, an $F_{*}^{1}$ contains at most one double point and a simple point of $F_{*}^{1}$ is an ordinary, inflection or cusp point; cf. [1] 1.4 and [2]. If $C(M)$ is a line [point], then $C$ is considered to have order one [two].

$\Gamma$ is of order $k$ if $k$ is the supremum of the number of points of $\Gamma$, counting multiplicities on each $C_{\lambda}$, lying on any line not in $\Gamma$. If $k=1$, then $\Gamma$ is a straight line. If $k=2$, then $\Gamma$ is an $S^{1}$ or an isolated point or a pair of distinct lines. If $k=3$, then $\Gamma$ is (i) an $F_{*}^{1}$ plus possibly an $S^{1}$ or an isolated point either disjoint from $F_{*}^{1}$ or (ii) the union of a line and a $\Gamma^{\prime}$ of order two. We denote a $\Gamma$ of order three satisfying (i) by $F^{1}$.

2. A surface of order three $\boldsymbol{F}$ in $P^{3}$ is a compact and connected set such that every intersection of $F$ with a plane is a curve of order $\leq 3$ and some plane intersection is an $F^{1}$.

Let $F$ be a surface of order three; $p \in F$. Let $\alpha$ denote a plane through $p$.

Received by the editors March 2, 1978 and, in revised form, September 7, 1978. 
Then $p$ is regular in $F[\alpha \cap F]$ if there is a line $N$ in $P^{3}[\alpha]$ such that $p \in N$ and $|N \cap F|=3$; otherwise, $p$ is irregular in $F[\alpha \cap F]$. If $\alpha \cap F$ is an $F^{1}$, then there is at most one irregular point $v$ in $\alpha \cap F$ and such a $v$ is a cusp, double point or isolated point. Finally,

$$
l(p, \alpha)=|\{L \subset \alpha \mid p \in L \subset F\}| \leq l(\alpha)=|\{L \subset \alpha \mid L \subset F\}| \leq 3 .
$$

A line $T$ is a tangent of $F$ at $p$ if $T$ is the tangent of some $C_{\lambda}$ at $m ; p=C_{\lambda}(m) \subset C_{\lambda}(M) \subset F$. Let $\tau(p)$ be the set of tangents of $F$ at $p$. Then $p$ is differentiable if $p$ is regular in $F$ and $\tau(p)$ is a plane $\pi(p)$; otherwise, $p$ is singular.

Henceforth, we assume that every regular $p$ in $F$ is differentiable and $\pi(p)$ depends continuously on $p$.

Let $p$ be differentiable. Then $p \in T \subset \pi(p)$ implies that either $T \subset F$ or $|T \cap F| \leq 2$. Thus $l(p)=|\{L \subset F \mid p \in L\}|=l(p, \pi(p))$ and $p$ is irregular in $\pi(p) \cap F$. If $l(p)=0$, then $p$ is an isolated point, cusp or double point of $\pi(p) \cap F$ and we call $p$ elliptic, parabolic or hyperbolic respectively.

Let $\mathscr{F}$ be a closed connected subset of $S^{1}$ or $F_{*}^{1}$. If the end points of $\mathscr{F}$ are distinct [equal], then $\mathscr{F}$ is a subarc [subcurve]. A subarc of $F_{*}^{1}$, containing only ordinary points in its interior, is of order two.

Let $p$ be regular in $F$. Let $\mathscr{F}(p)$ be the set of all subarcs $\mathscr{F}$ of order two such that $p \in \mathscr{F} \not \subset \pi(p) ;\left\{\mathscr{F}_{1}, \mathscr{F}_{2}\right\} \subset \mathscr{F}(p)$. Then $\mathscr{F}_{1}$ and $\mathscr{F}_{2}$ are $p$-compatible if there is a $\beta \subset P^{3} \backslash\{p\}$ and an open neighbourhood $U(p)$ of $p$ in $P^{3}$ such that $U(p) \cap$ $\left(\mathscr{F}_{1} \cup \mathscr{F}_{2}\right)$ is contained in a closed half-space of $P^{3}$ bounded by $\pi(p)$ and $\beta$; otherwise, $\mathscr{F}_{1}$ and $\mathscr{F}_{2}$ are $p$-incompatible.

A pair of subarcs $\mathscr{F}$ and $\mathscr{F}^{\prime}$ are compatible [incompatible] if there is a $p \in \mathscr{F} \cap \mathscr{F}^{\prime}$ such that $\left\{\mathscr{F}, \mathscr{F}^{\prime}\right\} \subset \mathscr{F}(p)$ and $\mathscr{F}$ and $\mathscr{F}^{\prime}$ are $p$-compatible $[p-$ incompatible]. We consider a subcurve of order two as an element of $\mathscr{F}(p)$ if it contains a subarc $\mathscr{F}$ such that $p \in \mathscr{F} \in \mathscr{F}(p)$.

3. We assume that $\boldsymbol{F}$ is non-ruled; that is, $F$ is not generated by lines. Then $l(F)=\left|\left\{l \subset P^{3} \mid L \subset F\right\}\right|<\infty$ and $F$ contains at most four irregular points; cf. [3]. We denote by $E, I$ and $H$ : the set of elliptic, parabolic and hyperbolic points of $F$ respectively. We shall prove that for any (non-ruled) $F: H \neq \phi, I \neq \phi$ implies that $E \neq \phi, E$ is open and $I$ is nowhere dense in $F$.

By way of preparation, we have the following remarks:

3.1 Let $L \subset F$ and $p \in F \backslash L$ such that $\langle L, p\rangle \cap F$ consists of $L$ and an $S^{1}$. We denote this $S^{1}$ by $S^{1}(L, p)$.

3.2 If a plane section of $F$ is of order two, then it consists of a pair of lines. ([1], 2.2.3.)

3.3 If $p$ is regular in $F$ and isolated in $\alpha \cap F$, then $p \in E$ and $\alpha=\pi(p)$. ([1], 2.3.7.)

3.4 Let $p$ be regular in $F, l(p)=0$. Then $p \in H$ if and only if there exist incompatible $\mathscr{F}$ and $\mathscr{F}^{\prime}$ in $\mathscr{F}(p)$ with $p \in \operatorname{int}(\mathscr{F}) \cap \operatorname{int}\left(\mathscr{F}^{\prime}\right)$. $([1], 2.5 .7)$ 
3.5 Let $\mathscr{F}^{\prime} \in \mathscr{F}(p)$ for each $p \in \mathscr{F}^{\prime}$. Let $L \subset F$ such that $L \not \subset\left\langle\mathscr{F}^{\prime}\right\rangle$ and $S^{1}(L, p) \in$ $\mathscr{F}(p)$ for each $p \in \mathscr{F}^{\prime}$. Then $\mathscr{F}^{\prime}$ and $S^{1}(L, p)$ are either compatible for all $p \in \mathscr{F}^{\prime}$ or incompatible for all $p \in \mathscr{F}^{\prime}$. ([1], 2.5.8.)

3.6 Let $p_{\lambda}$ be a sequence converging to a differentiable $p$. If $p_{\lambda} \in I[E]$ for each $\lambda$, then $l(p)=0$ implies that $p \in I[E \cup I]$ and $\pi(p) \cap F=L \cup S^{1}$ implies that $L \cap S^{1}=\{p\}$. ([1], 2.4.6 and 2.4.9.)

3.7. Lemma. Let $G$ be an open region in $F$ such that $\alpha_{0} \cap \bar{G}=\phi$ for some $\alpha_{0}, b d(F \backslash G)=b d(G),\langle b d(G)\rangle$ is a plane and each $p \in G$ is regular in $F$. Then $G \cap E \neq \phi$.

Proof. We note that any line in a plane $\left\langle F_{*}^{1}\right\rangle$ meets $F_{*}^{1}$ and thus, any line in $P^{3}$ meets $F$ by 3.2.

Let $p \in G$ and put $L=\alpha_{0} \cap\langle b d(G)\rangle$. Then $L \cap \bar{G}=\phi$ implies that $L \cap(F \backslash \bar{G}) \neq \phi$ and $\langle L, p\rangle \cap G$ is an $S^{1}$ or an isolated point of $\langle L, p\rangle \cap F$. Obviously, $\alpha_{0} \cap \bar{G}=\phi$ yields that there is a $p_{0} \in G$ such that $\left\langle L, p_{0}\right\rangle \cup G=\left\{p_{0}\right\}$. Then $p_{0} \in E$ and $\pi\left(p_{0}\right)=\left\langle L, p_{0}\right\rangle$ by 3.3 .

We note that $\bar{E} \cap H=\phi$ and $\bar{I} \cap(E \cup H)=\phi$ by 3.6. It is clear that a limit of hyperbolic points may be parabolic but not elliptic. Thus, $E$ is open in $F$ and

$$
\{p \in \bar{E} \cap \bar{H} \mid l(p)=0 \text { and } p \text { is regular in } F\} \subseteq I .
$$

3.8. Theorem. If $l(F)>0$, then $I$ is nowhere dense in $F$ and

$$
I=\{p \in \bar{E} \cap \bar{H} \mid l(p)=0 \text { and } p \text { is regular in } F\} .
$$

Proof. Let $L \subset F$ and $p_{0} \in I$. Then there is a $T \subset \pi\left(p_{0}\right)$ such that $T \cap F=\left\{p_{0}\right\}$. Let $T \subset \beta \neq \pi\left(p_{0}\right)$. Then $l\left(p_{0}\right)=0, T \cap F=\left\{p_{0}\right\}$ and 3.2 imply that $\beta \cap F$ is an $F^{1}$ with $p_{0}$ as an inflection point. Thus there are $\mathscr{F}$ and $\mathscr{F}^{\prime}$ in $\mathscr{F}\left(p_{0}\right)$ such that $\mathscr{F} \cup \mathscr{F}^{\prime} \subset \beta$ and $\mathscr{F} \cap \mathscr{F}^{\prime}=\left\{p_{0}\right\}$. We note that $\mathscr{F}$ and $\mathscr{F}^{\prime}$ are incompatible and for $p$ close to $p_{0}$ in $\mathscr{F} \cup \mathscr{F}^{\prime}, p$ is regular in $F$. Since $l\left(p_{0}\right)=0$ and $T \cap L=\phi$, we may assume that $l(p)=0$ and $(\beta \cap \pi(p)) \cap L=\phi$ for each $p \in \mathscr{F} \cup \mathscr{F}^{\prime}$. Then $\langle L, p\rangle \cap F=L \cup S^{1}(L, p)$ for each $p \in \mathscr{F} \cup \mathscr{F}^{\prime}$ by 3.3 .

(i) Since $\mathscr{F}$ and $\mathscr{F}^{\prime}$ are incompatible, $S^{1}\left(L, p_{0}\right) \in \mathscr{F}\left(p_{0}\right)$ implies that $S^{1}\left(L, p_{0}\right)$ and say $\mathscr{F}$ are incompatible. By 3.5, $S^{1}(L, p)$ and $\mathscr{F}$ are incompatible for each $p \in \operatorname{int}(\mathscr{F})$. Thus $p_{0} \in \bar{H}$ by 3.4 .

(ii) Since $p_{0} \in I$,

$$
\pi\left(p_{0}\right) \cap F=\mathscr{F}_{1} \cup \mathscr{F}_{2}
$$

where $\mathscr{F}_{1}\left[\mathscr{F}_{2}\right]$ is a subarc of order two, $p_{0} \in \mathscr{F}_{1} \cap \mathscr{F}_{2}$ and $\left|\mathscr{F}_{1} \cap \mathscr{F}_{2}\right|=2$. Let $p_{\lambda}$ in $\operatorname{int}(\mathscr{F})$ converge to $p_{0}$. Since $p_{\lambda} \in H$ for each $\lambda$,

$$
\pi\left(p_{\lambda}\right) \cap F=\mathscr{L}_{\lambda} \cup \mathscr{F}_{1, \lambda} \cup \mathscr{F}_{2, \lambda}
$$

where $\mathscr{L}_{\lambda}$ is a subcurve of order two, $\mathscr{F}_{1, \lambda}\left[\mathscr{F}_{2, \lambda}\right]$ is a subarc of order two, $\mathscr{L}_{\lambda} \cap\left(\mathscr{F}_{1, \lambda} \cup \mathscr{F}_{2, \lambda}\right)=\left\{p_{\lambda}\right\}, p_{\lambda} \in \mathscr{F}_{1, \lambda} \cap \mathscr{F}_{2, \lambda}$ and $\left|\mathscr{F}_{1, \lambda} \cap \mathscr{F}_{2, \lambda}\right|=2$. We note that as 
$p_{\lambda}$ tends to $p_{0}$,

$$
\lim \pi\left(p_{\lambda}\right) \cap F=\pi\left(p_{0}\right) \cap F
$$

and $\lim \mathscr{L}_{\lambda}$ is a closed curve of order $\leq 2$. It is easy to check that

$$
\lim \mathscr{L}_{\lambda}=\left\{p_{0}\right\} \quad \text { and } \quad \lim \mathscr{F}_{1, \lambda} \cup \mathscr{F}_{2, \lambda}=\mathscr{F}_{1} \cup \mathscr{F}_{2}
$$

Since $p_{0}$ is parabolic, we can describe a sufficiently small neighbourhood of $p_{0}$ in $F$. In particular, it is easy to check that (for $p_{\lambda}$ sufficiently close to $p_{0}$ ) $\mathscr{L}_{\lambda}$ is the boundary of an open region $F\left(\mathscr{L}_{\lambda}\right) \subset F$ such that $b d\left(F \backslash F\left(\mathscr{F}_{\lambda}\right)\right)=$ $b d\left(F\left(\mathscr{L}_{\lambda}\right)\right)$ and $\lim \overline{F\left(\mathscr{L}_{\lambda}\right)}=\left\{p_{0}\right\}$. Finally, $l\left(p_{0}\right)=0$ and $p_{0}$ regular in $F$ imply that $F\left(\mathscr{L}_{\lambda}\right)$ satisfies 3.7 for $p_{\lambda}$ sufficiently close to $p_{0}$. Hence, $F\left(\mathscr{L}_{\lambda}\right) \cap E \neq \phi$ and $p_{0} \in \bar{E}$.

3.9. ThEOREM. If $l(F)>0$, then $F$ contains hyperbolic points.

Proof. We note that $l(\beta)=0$ implies that $\beta \cap F$ contains an inflection point by 3.2. As $l(F)<\infty$, the set

$$
\mathscr{Q}=\{q \in F \mid q \text { is an inflection point }\}
$$

is infinite. If $q \in \mathscr{Q}$ and $l(q)=0$, then $q \in I \cup H$. Since $I \neq \phi$ implies that $\bar{E} \cap \bar{H} \neq \phi$ (and thus $E \neq \phi \neq H$ ) by 3.8, we obtain that $q \in I \cup H$ yields that $H \neq \phi$.

Suppose that $H=\phi$. Then $l(q)>0$ for each $q \in \mathscr{Q}$ and there is an $L \subset F$ such that $L \cap \mathscr{Q}$ is an infinite set.

CASE 1. There is an $M \subset F$ such that $L \cap M=\phi$.

Let $q \in L \cap 2$. Then $\pi(q)=\left\langle L, T_{q}\right\rangle$ where $T_{q} \cap F=\{q\}$. As $\pi(q) \cap M \not \subset L$, this implies that either $\langle q, \pi(q) \cap M\rangle \subset F$ or $\pi(q) \cap F$ consists of $L$ and an $S^{1}$ where $\left|L \cap S^{1}\right|=2$ and $q \in L \cap S^{1}$. In the latter case, 3.6 clearly implies that $q \in \bar{H}$ and $H \neq \phi$; a contradiction. Thus $\langle q, \pi(q) \cap M\rangle \subset F$ for each $q \in L \cap \mathscr{Q}$. Then $l(F)<$ $\infty$ implies that $|L \cap \mathscr{Q}|<\infty$; a contradiction.

CASE 2. Every $M \subset F$ meets $L$.

Clearly, there is a point $q_{1} \in \mathscr{Q} \backslash L$. Thus there is an $L_{1} \subset F$ such that $q_{1} \in L_{1}$ and $L_{1} \cap L$ is a point $v \neq q_{1}$.

If $M \subset F$ such that $M \cap L_{1}=\phi$, then $M_{1}=\left\langle q_{1}, \pi\left(q_{1}\right) \cap M\right\rangle \subset F$ by the preceding. Since $v \notin M_{1}$ and $M_{1} \cap L \neq \phi$, we obtain that $M_{1} \subset\left\langle L, L_{1}\right\rangle$. Then $\pi\left(q_{1}\right) \cap F=M_{1} \cup L_{1} \cup L$ where $M_{1} \cap L_{1} \cap L=\phi$ implies that $q_{1} \notin 2$; a contradiction. Thus, every line in $F$ passes through $v$.

Let $v \in \beta$. Then either $l(\beta)>0$ or $(l(\beta)=0, \beta \cap \mathscr{Q}=\{v\}$ and) $\beta$ contains an irregular point of $F$. Since $F$ contains a finite number of lines and irregular points, this is a contradiction. 
4. In each of the following examples, $F$ contains exactly one irregular point $v$ and one line $L$. Recall that $\tau(v)$ is the set of tangents of $F$ at $v$.

Let $P^{3}$ be suitably coordinatized.

The surface $F$ defined by

$$
0=x_{0}^{3}-\left(x_{1}^{2}+x_{2}^{2}\right) x_{3} \quad\left(L \equiv x_{0}=x_{3}=0, v \equiv(0,0,0,1)\right)
$$

contains neither elliptic nor parabolic points. $\tau(v)$ is a line $T \equiv x_{1}=x_{2}=0$ and $v$ is the cusp [isolated point] of $\beta \cap F$ if $T \subset \beta[\beta \cap T=\{v\}]$.

The surface $F$ defined by

$$
0=x_{0}^{3}-\left(x_{1}^{2}+x_{2}^{2}-x_{0}^{2}\right) x_{3} \quad\left(L \equiv x_{0}=x_{3}=0, v \equiv(0,0,0,1)\right)
$$

contains elliptic but not parabolic points. $\tau(v)$ is a cone of order two with vertex $v$ and $v \in T \subset \tau(v)$ if and only if $T \cap F=\{v\}$. If $\beta \cap \tau(v)=\{v\}$, then $v$ is the isolated point of $\beta \cap F$; otherwise, $v$ is the cusp or the double point of $\beta \cap F$. Finally, $E$ and $H$ are both connected with $\bar{E}=E \cup\{v\}$ and $\bar{H}=$ $H \cup L \cup\{v\}$.

The surface $F$ defined by

$$
0=x_{0}^{3}+x_{1} x_{2}^{2}+x_{1}^{2} x_{3} \quad\left(L \equiv x_{0}=x_{1}=0, v \equiv(0,0,0,1)\right)
$$

contains parabolic points, $\tau(v) \equiv x_{1}=0$ and $\beta \cap L=\{v\}$ implies that $v$ is the cusp of $\beta \cap F$. In this case; $I=S^{1}(L, \bar{p}) \backslash\{v\}$ where $\bar{p} \equiv(0,1,0,0)$ or equivalently $I \equiv 0=x_{0}=x_{2}^{2}+x_{1} x_{3}, E$ is connected with $\bar{E}=E \cup I \cup\{v\}$ and $\bar{H}=H \cup I \cup L$.

\section{REFERENCES}

1. T. Bisztriczky, Surfaces of order three with a peak. I., J. of Geometry, Vol. 22 (1) (1978), 55-83.

2. O. Haupt and H. Künneth, Geometrische Ordnungen, Springer-Verlag, Berlin, 1967.

3. A. Marchaud, Sur les surfaces du troisième ordre de la Géométrie finie, J. Math. Pur. Appl. 18, (1939), 323-362.

4. P. Scherk, Über differenzierbare Kurven und Bögen. I. Zum Begriff der Charakteristik, Časopis Pěst. Mat. 66 (1937), 165-171.

Department of Mathematics and Statistics

THE UNIVERSTTY OF CALGaRY,

Calgary, Alberta. T2N 1N4 